\title{
SiPHonaptères de TANZANIE. Description de deUX taXa et COMPLÉmentS À LA DESCRIPTION DE CTENOPHTHALMUS COPHURUS heMINGWAYI HUBBARD, 1963 (SiPHONAPTERA : CTENOPHTHALMIDAE)
}

\author{
LAUDISOIT A.$^{*, * *, * * *} \&$ BEAUCOURNU J.-C.****,******
}

Summary: SIPHONAPTERA FROM TANZANIA. DESCRIPTION OF TWO TAXA AND COMPLEMENTARY DESCRIPTION OF CTENOPHTHALMUS COPHURUS HEMINGWAYI HubBaRD, 1963 (Siphonaptera: CTENOPHTHALMidaE)

Two new taxa, Ctenophthalmus (Ethioctenophthalmus) iraqwi n. sp. and C. (E.) evidens derocki n. ssp. are described and a complementary description is given for C. (E.) cophurus

hemingwayi Hubbard, 1963, all those taxa belonging to the Tanzanian flea fauna.

KEY WORDS : Siphonaptera, Tanzania, Ctenophthalmus (Ethio.) iraqwi n. sp., Ctenophthalmus (Ethio.) evidens derocki n. ssp., Ctenophthalmus (Ethio.) cophurus hemingwayi.

\section{INTRODUCTION}

T ors de recherches sur les vecteurs dans les foyers pesteux de Tanzanie, l'un de nous (A.L.) avait $\checkmark$ récolté deux espèces nouvelles (C. teucqae et C. kemmelberg) (Laudisoit \& Beaucournu, 2007a, b) appartenant au genre et au sous-genre Ctenophthalmus (Ethioctenophthalmus). La description de la première a permis, de plus, la mise en évidence d'une sousespèce nouvelle, car mal identifiée, déposée au Natural History Museum (Londres) et qui fut étudiée en même temps que notre matériel : il s'agit de $C$. (E.) teucqae shumeensis $\mathrm{n}$. ssp. (Laudisoit \& Beaucournu, 2007a) provenant également du district de Lushoto en Tanzanie. De nouvelles collectes effectuées dans un second district de peste endémique plus septentrional (Mbulu, région du Manyara) ont livré, toujours dans les mêmes genres et sous-genres, une espèce et une sous-espèce nouvelles; par ailleurs, C. (E.) cophurus hemingwayi Hubbard, 1963, a été retrouvée et est redécrite ici.

* CERVA-CODA, Bactériologie générale, 99, Groeselegen, 1180 Uccle, Belgique; ** Université d'Anvers, Unité EVOBIO, 171, Groenenborgerlaan, 2020 Antwerpen, Belgique; *** Université de Liège, Unité de recherches zoogéographiques, Faculté des sciences, 27, Boulevard du Rectorat, Bâtiment B22 Botanique, 4000 Liège (Sart-Tilman), Belgique.

***** Laboratoire de Parasitologie et Zoologie appliquée, Faculté de Médecine, 2, Avenue Léon-Bernard, 35043 Rennes cedex, France;

**:**** Institut de Parasitologie de l'Ouest, Faculté de Médecine, même adresse.

Correspondance : Jean-Claude Beaucournu.

E-mail : jeanclaude.beaucournu@gmail.com

\section{Résumé :}

Deux nouveaux taxa, Ctenophthalmus (Ethioctenophthalmus) iraqwi n. sp. et C. (E.) evidens derocki n. ssp. sont décrits et des compléments de description sont donnés pour C. (E.) cophurus hemingwayi Hubbard, 1963, tous ces taxa concernant la faune de Tanzanie.

MOTS CLÉS : Siphonaptera, Tanzanie, Ctenophthalmus (Ethio.) iraqwi n. sp., Ctenophthalmus (Ethio.) evidens derocki n. ssp., Ctenophthalmus (Ethio.) cophurus hemingwayi.

\section{RÉSULTATS}

\section{CTENOPHTHALMUS \\ (ETHIOCTENOPHTHALMUS) IRAQWI N. SP.}

Matériel de description : mâle holotype sur Lophuromys sp. ( $\mathrm{n}^{\circ}$ TE 5693), région de Manyara, district de Mbulu, village de Tumati, localité de Mongahay (S $4^{\circ} 03^{\prime}$ 58.1"; E $\left.35^{\circ} 27^{\prime} 22.7^{\prime \prime}\right)$, en forêt primaire d'altitude, alt. 2210 m, 14 février 2008 (les analyses de séquences de cytochrome-c rapprochent l'espèce de Lophuromys du district de Mbulu à la branche de Lophuromys dudui, décrit de la région de Kisangani en République Démocratique du Congo (Verheyen E., comm. pers.); femelle allotype, sur le même individu-hôte que l'holotype; deux femelles paratypes sur Lophuromys sp. ( ${ }^{\circ}$ TE 5985), même localisation mais en lisière de forêt, 14 mars 2007; une femelle paratype sur Lophuromys sp. (n TE 5699), même localisation que les types, même date ; une femelle paratype sur Lophuromys sp. ( $\mathrm{n}^{\circ}$ TE 5743), même localisation que les types, 16 février 2008; une femelle paratype sur Lophuromys sp. ( $\mathrm{n}^{\circ} \mathrm{TE}$ 5704), même localité et village que les types mais en champs et jachères, 14 février 2008; un mâle paratype sur Lophuromys sp. (n TZ 22519), région du Manyara, district de Mbulu, village de Tumati, localité de Mongahay (S 4 03 ' 57.8'; E $35^{\circ} 27^{\prime} 23.3^{\prime \prime}$ ), en forêt primaire d'altitude, alt. $2212 \mathrm{~m}, 9$ février 2009. Tous les spécimens ont été collectés en 2007 et 2008 par l'équipe du Dr Rhodes Makundi (Pest Management Centre, Morogoro, Tanzanie) et en 2009 par la même équipe et A.L. 
Dépôt des types : holotype, allotype et trois femelles paratypes dans les collections d'entomologie du Muséum royal de l'Afrique Centrale de Tervuren (Belgique); le mâle paratype et deux femelles paratypes dans les collections du deuxième auteur, ultérieurement déposées au Laboratoire d'entomologie du Muséum national d'Histoire naturelle de Paris (France).

Derivatio nominis : cette espèce est nommée en hommage aux hommes et aux femmes Iraqw, peuple très peu connu, effacé et pourtant majoritaire, vivant dans le district de Mbulu, parlant une langue kouchitique d'origine nilo-saharienne interrompue de "clics", et qui sont arrivés il y a près de 2000 ans dans la région.

\section{Description}

Capsule céphalique (figure 1). La partie antérieure, ou frontale, est nettement divisée en deux zones de pigmentation différentes, la plus dorsale étant plus foncée que la plus basale. Des micropores sont seuls présents dans la zone dorsale; on ne trouve que des soies et des micro-soies dans la basale. CEil peu pigmenté, à contours flous. Area fusoria non visible chez le mâle, grande chez la femelle. Cténidie génale montrant un diastème entre la première et la deuxième dent, celleci est arrondie à l'apex; la troisième s'implante, en vue transversale, au même endroit que la seconde qui recouvre ainsi la base de la troisième qui est longue et acuminée. Ce recouvrement est moins marqué chez les femelles. Palpe labial un peu plus long que la moitié de la coxa I.

Thorax. Prothorax montrant une cténidie de 16 épines, précédée d'un rang de six longues soies. Le bord libre du segment est un peu plus court que les épines dorsales de la cténidie. Mesothorax portant un rang de six petites soies, puis cinq fortes et plus longues et, enfin, deux pseudo-setae. Metathorax : trois rangs de soies, le premier vestigial de deux à trois petites soies, le deuxième de six soies et le troisième de six soies fortes. Métépiméron : un rang antérieur, irrégulier, de six soies, suivies de trois soies plus fortes. Toutes sont implantées au-dessous du spiracle, cordiforme et à atrium nu. Métatibia portant sept encoches postérieures avec de fortes soies, la plus longue étant plus courte que le premier tarsomère. Trois soies latérales sur le distitarsomère.

Abdomen (segments non génitaux). Le bord postérieur des tergites I à IV porte des spinules : $1-1$ ou $2-1$ - 1. Rangée principale de huit soies, la plus basse étant insérée au-dessous du spiracle. Celui-ci est pyriforme et son atrium nu, sauf évidemment celui du tergite VIII. Tergite VII portant les trois soies anté-sensiliales classiques de ratio $0,3-1-0,3$ chez le mâle et $0,3-1-0,6$ chez la femelle. Dans les deux sexes, elles sont précédées de quatre soies courtes et fortes. Sternites : la sétation chez le mâle, du st. II au st. VII est de : $1-6$ (en plage) -6 (sur deux rangs) -6 (sur deux rangs)
-7 (sur deux rangs) $-8-8$; chez la femelle, 2 (très petites) $-7-8-9-5-13-6$ marginales et fortes, surmontées de quatre plus petites.

Abdomen (segments génitaux du mâle) (figure 2) : sternite VIII montrant un petit lobule apical et portant 12 à 13 soies. Segment IX : manubrium large; pas d'area fusoria; processus basimeris plus ou moins quadrangulaire, pigmenté, portant en diagonale quatre longues et fortes soies. Soie acétabulaire plus courte que la largeur, à ce niveau, du basimère. Bord apical sans tubercules, mais avec deux "bourrelets" marginaux fortement sclérotisés et pratiquement jointifs; à la base antérodorsale du premier, on note la soie apicale du basimère qui est ici petite et fine et termine vers le haut une rangée de soies identiques de la marge antérieure. Processus telomeris (ou télomère) ne montrant pas la tétrade de soies classique. Apex avec quatre sensillae. Le télomère est trois fois plus long que large à sa plus grande largeur qui est à l'union du premier et du deuxième tiers de sa longueur. Fovea longue, peu large, "appuyée" sur le deuxième bourrelet du basimère. Sternite : apex large, bras apical fin et rectiligne, bras distal mesurant la moitié de la longueur du bras apical, large et discrètement courbé. À son extrémité, une petite encoche est présente et une rangée ventromarginale de six à sept petites soies fines. Le bord ventral est dédoublé en gouttière sur presque toute sa longueur.

Phallosome (figure 3). Nous ne figurons que l'aedeagus. Lobe médio-dorsal peu développé, le sclérite de Ford en suit la marge jusqu'au tubus interior qui est grand et net. Hamulus long et très acuminé. Lamella grande avec un petit repli ( $c f$. flèche figure 3) à son extrémité antérieure.

Abdomen (segments génitaux de la femelle) (figure 4). Sternite VII montrant une large concavité à sa marge postérieure qui est pigmentée dans sa moitié basale. Tergite VIII sans soie en avant du spiracle, avec un lobe apical large et arrondi sur sa marge postérieure. Sternite VIII large et doucement pigmenté.

Spermathèque et ducti (figure 4) : bulga asymétrique, et hilla longue et courbée. Ductus bursae fin et doucement arqué.

Dimensions (insectes montés) : mâle holotype 2,1 mm (paratype 2,5); femelle allotype 2,3 (paratypes 2,3 à 2,5, moyenne 2,35).

\section{Discussion}

La morphologie de l'aedeagus écarte, à elle seule, l'attribution aux deux autres sous-genres afrotropicaux, Idioctenophthalmus et Geoctenophthalmus. Si C. iraqwi appartient de toute évidence à Ethioctenophthalmus Hopkins \& Rothschild, 1966, il ne trouve sa place dans aucun des groupes d'espèces définis par ces auteurs. L'œil peu pigmenté, la brièveté relative du palpe labial, la sétation du tarse III, comme celle du 


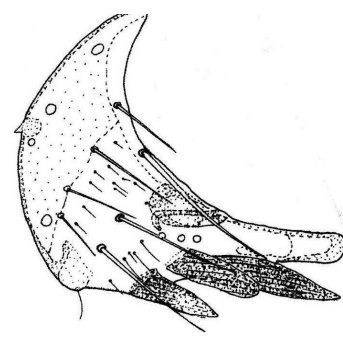

1
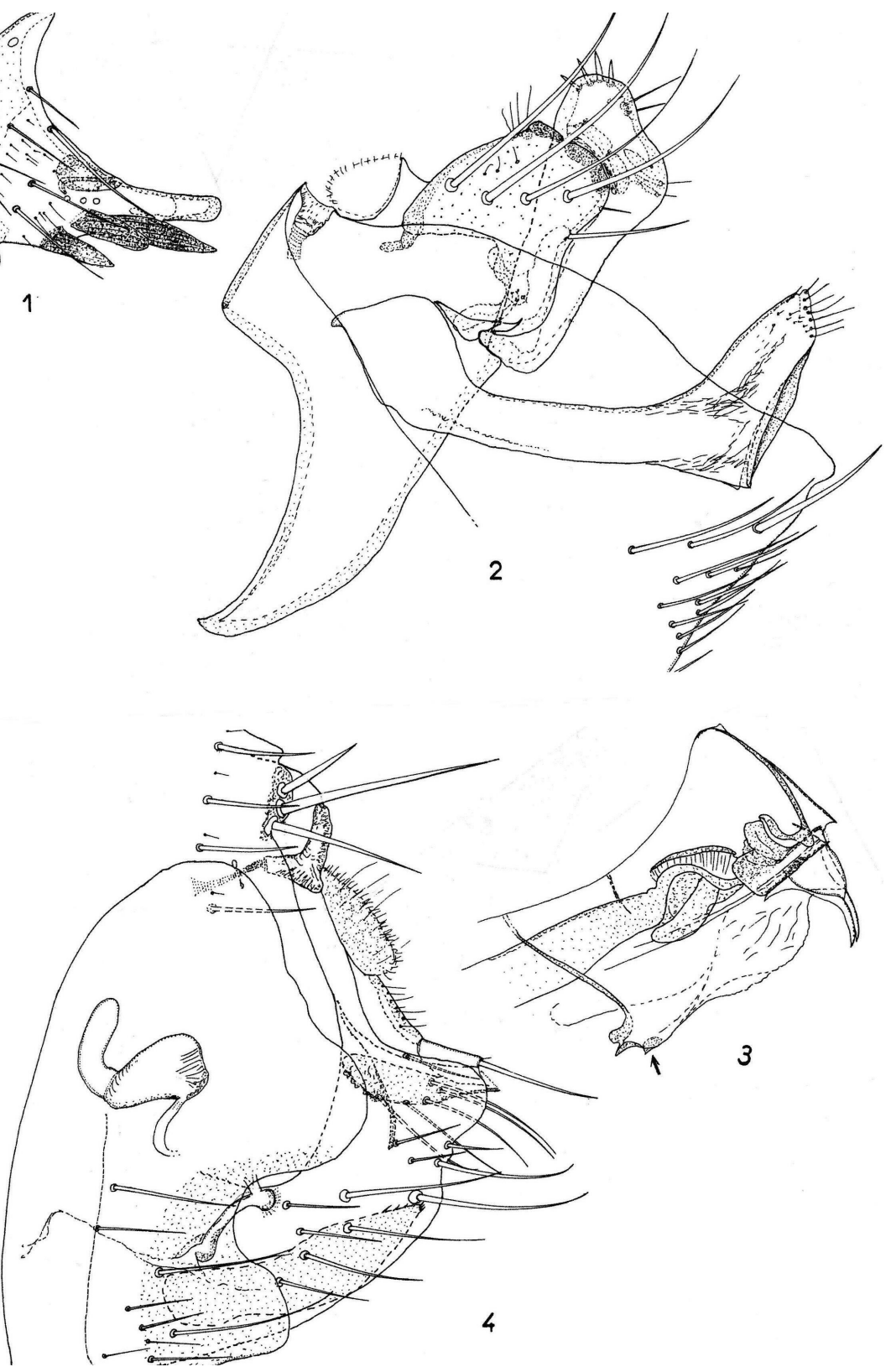

Figs 1-4. - Ctenophthalmus (Ethioctenophthalmus) iraqwi n. sp. 1 : holotype, partie frontale de la capsule céphalique; 2 : holotype, segments VIII et IX; 3 : holotype, aedeagus; 4 : allotype, sternite VII et segment VIII, spermathèque et ducti. distitarsomère, l'absence d'area fusoria, la sétation du processus basimeris... privilégient tantôt un groupe, tantôt un autre. Nous préférons donc le classer, provisoirement, dans les "incertae sedis".

\section{CTENOPHTHALMUS (ETHIO.) EVIDENS DEROCKI N. SSP.}

Matériel de description. Holotype et allotype sur Lophuromys $\mathrm{sp}$. ( $\mathrm{n}^{\circ} \mathrm{TZ} 22537$ ), région du Manyara, district de Mbulu, village de Tumati, localité de Mongahay (S $4^{\circ} 03^{\prime}$ 57.8'; E $35^{\circ} 27^{\prime} 23.3^{\prime \prime}$ ), en forêt naturelle primaire d'altitude, alt. $2212 \mathrm{~m}, 10$ février 2009; deux femelles paratypes sur Lophuromys sp. ( ${ }^{\circ} \mathrm{TZ} 22536$ ), même endroit, même date; un mâle paratype sur
Lophuromys sp. (n TZ 22497), même endroit mais village de Nahasey, localité de Ayabalangw (S 3 54' 20.5"; E $35^{\circ} 37^{\prime}$ 42.6”), alt. 1884 m, 7 février 2009; un mâle paratype sur Lophuromys sp. (n TZ 22520), même localisation que les types.

Dépôt des types, comme ci-dessus.

Derivatio nominis: taxon dédié à une famille amie au nom évoquant les cirques rocailleux qui dominent les hauts plateaux des montagnes d'Afrique de l'Est où sévit encore la peste bubonique.

Description

Seuls les segments génitaux seront étudiés, puisqu'il s'agit d'une sous-espèce. Avant la récolte de cette nouvelle sous-espèce, six taxa étaient décrits pour 
C. evidens. Par ordre chronologique, ce sont C. evidens evidens Jordan, 1929, C. e. modicus Jordan, 1933, C. e. elgonensis Jordan, 1938, C. e. wilkesi Hubbard, 1963, C. e. convexus Smit, 1975 et C. e. abyssinicus Beaucournu, 1991. La répartition inclut donc la RDC, le Kenya, la Tanzanie, l'Ouganda et nettement plus septentrionale, l'Éthiopie. C. evidens derocki ssp. n. constitue la deuxième sous-espèce connue de Tanzanie. Segments génitaux du mâle (figure 5). Sternite VIII montrant une petite concavité sur sa marge ventrale; sur celle-ci, une plage de six soies dont deux longues. Segment IX : tergite montrant une area fusoria. Le processus basimeris est plus ou moins quadrangulaire, environ deux fois plus haut que large, mais se rétrécissant vers l'apex. Une rangée marginale sur le bord antéro-supérieur de six longues soies, touchant le bord apical et pouvant masquer la soie apicale du basimère. Deux ou trois longues soies sont implantées, en "bandeau" sur la face externe du processus. La soie acétabulaire, insérée au tiers supérieur, est une fois et demie aussi longue que la largeur de celui-ci. Le bord apical est perpendiculaire à la marge antéro-supérieure; il montre deux tubérosités virtuelles en ce sens que leur individualité tient plus à leur forte pigmentation, qu'à un relief réel de ce bord. Processus telomeris deux fois et demi plus long que large, sa plus grande largeur étant au tiers apical. Quatre sensillae à l'angle antérosupérieur, puis une marge doucement concave, et fortement oblique vers le bas, allant jusqu'au lobe convexe du bord postéro-ventral : il porte la tétrade
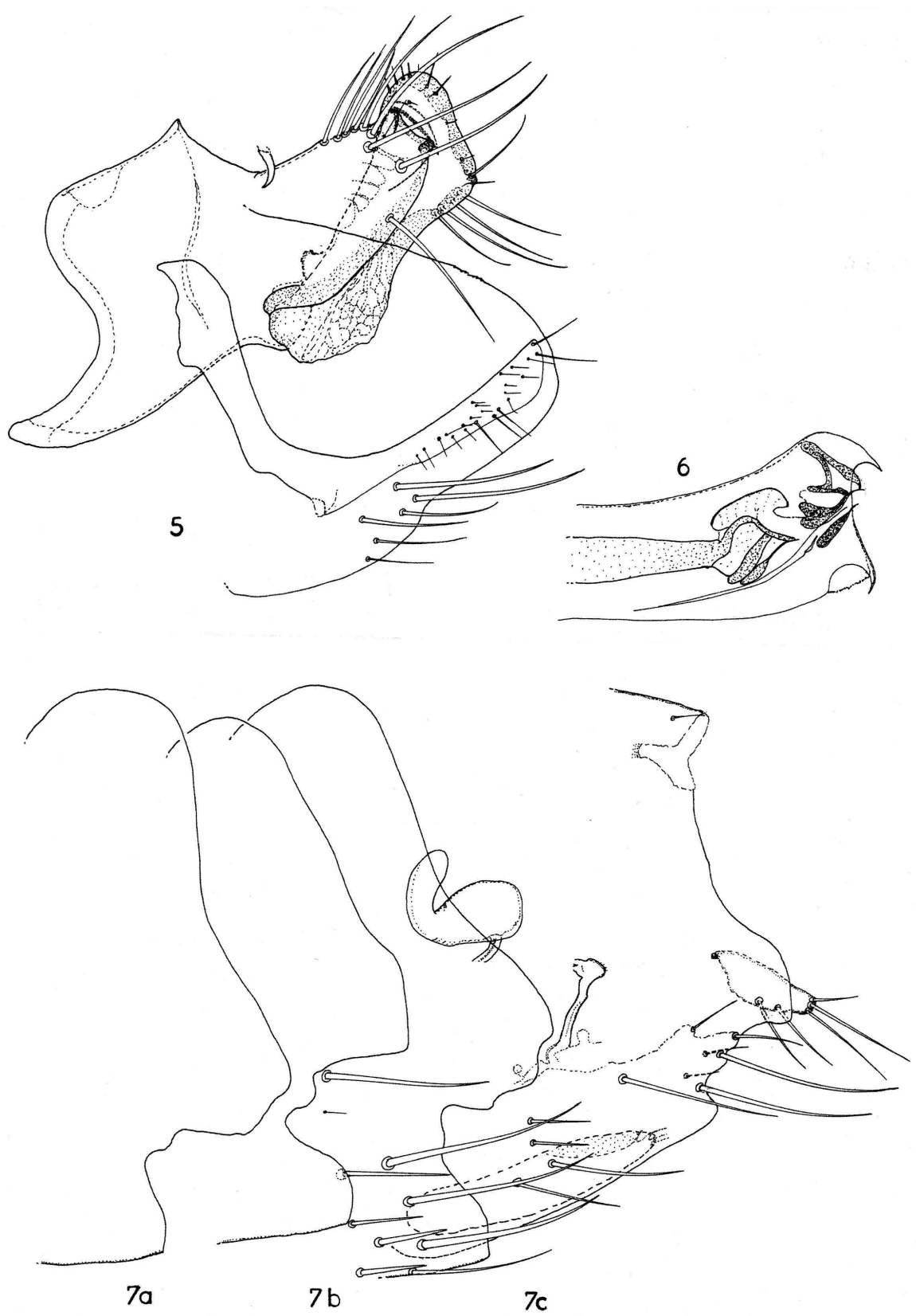

Figs 5-7. - Ctenophthalmus (Ethio.) evidens derocki n. ssp. 5 : holotype, segments VIII et IX; 6 : holotype, aedeagus; 7 : a et b, sternite VII de deux paratypes, c, Allotype, sternite VII et segment VIII, spermathèque et ducti. 
de soies classique. La moitié basale de ce bord est finement ornementée de ciselures irrégulières. Les deux bras, apical et distal, du sternite sont fins et de longueur équivalente. Ils ne semblent montrer aucun signe discriminant.

Phallosome (figure 6) : l'aedeagus est conforme à ceux des autres sous-espèces.

Segments génitaux de la femelle (figure $7 \mathrm{a}, \mathrm{b}, \mathrm{c}$ ). Ils paraissent peu ou non différenciables de ceux des autres sous-espèces où ils furent dessinés (evidens, convexus et abyssinicus) à l'exception de ceux de modicus. Le sternite VIII est toutefois différent de celui de la forme nominative.

\section{Discussion}

Ctenophthalmus (Ethio.) evidens derocki ssp. n. se séparera de toutes les autres sous-espèces, à l'exception d'abyssinicus, par la forme du basimère, et surtout du télomère : apex légèrement concave, oblique, convexité du lobe postérieur et concavité de la marge. La sousespèce abyssinicus, dont le télomère est assez proche, s'isolera par la forme générale du basimère aussi large dans sa partie inférieure que dans sa partie supérieure, une marge antéro-dorsale non perpendiculaire à la marge apicale, celle-ci n'étant pourvue que d'une seule "tubérosité" occupant les deux tiers de ce bord.

\section{CTENOPHTHALMUS (E.) COPHURUS HEMINGWAYI HUBBARD, 1963}

Compléments de description (figures 8-13)

Actuellement, quatre sous-espèces sont décrites: $C$. $c$. cophurus Jordan \& Rothschild, 1913, C. c. hemingwayi Hubbard, 1963, C. c. grzimeki Hubbard, 1964 et C. c. prolapsus Smit 1975. La sous-espèce "bemingwayi" n'était connue que par le seul matériel de description de Tanzanie : "off L. flavopunctatus margarettae Heller trapped along a creek bank several miles above the Forestry College north of Ngaramtoni Juu (Arusha) at an elevation of 7,000 feet on the south-west slope of Mt. Meru" (Hubbard, 1963). C. cophurus hemingwayi présente un caractère original, le bras distal du sternite IX, qui bien que minutieusement figuré par A. Smith (in : Hopkins \& Rothschild, 1966, fig. 453) est, comme souvent chez ce dessinateur trop pointilleux, noyé dans la surimpression de tous les plans anatomiques. Ce sternite a été négligé, à tort, par Smit (1975) qui ne redonne, pour "bemingwayi", qu'un dessin du tergite IX et précise "these two forms (la deuxième est schmiederi) appear to differ only in the shape of the télomère". Il figure le sternite IX de C. c. schmiederi mais, pour une raison inexplicable, pas celui de C.c. hemingwayi dont la morphologie, à l'étude minutieuse du dessin de A. Smith (op. cit.), n'a que peu de ressemblance avec celui de "schmiederi". Nous considérons que ce segment, en particulier, est suffisamment curieux pour être de nouveau étudié et ceci pourra faciliter l'identification des diverses sous-espèces de C. cophurus.

Matériel de comparaison. Deux femelles sur Lophuromys aquilus, Gologolo, site de Grewal (S 40 43' 29.6"; E $38^{\circ}$ 15' 04.9'), Tanzanie, alt. 1955 m, 18 juillet 2005; deux mâles, une femelle sur L. aquilus, même endroit, 19 juillet 2005; une femelle sur L. aquilus, même endroit, 20 juillet 2005; un mâle sur L. aquilus, même endroit, 21 juillet 2005.

Pour Wilson \& Reeder (1993), Lophuromys aquilus serait synonyme de L. flavopunctatus qui a la priorité, mais de nouveaux travaux séparent distinctement ces deux espèces et donnent leurs aires de distribution (Verheyen et al., 2007; Vrenchenko et al., 2007).

\section{Description}

Seuls, comme il est classique pour une sous-espèce, les segments génitaux VIII et IX et le phallosome, seront re-examinés chez le mâle. Les femelles de ce complexe ne semblent pas séparables (Hopkins \& Rothschild, 1966), en dépit de l'opinion de Hubbard (1963, inter alia). Smit (1963) ne connaissait que C. cophurus Jordan \& Rothschild, 1913, et n'a donc pas pu donner son avis dans sa Monographie du genre Ctenophthalmus. Quant à la femelle de C. c. prolapsus Smit, 1975, elle reste inconnue.

Segments modifiés du mâle. Tergite VIII : à titre documentaire et pour illustrer notre récent travail sur les spiracles chez les Siphonaptères (Beaucournu \& Laudisoit, 2009) nous figurons celui de C. c. hemingwayi comparé à celui de C. kemmelberg Laudisoit \& Beaucournu, 2007b, deux taxa vivant en syntopie (figures 8 et 14) et montrant cependant une morphologie différente du spiracle. Le sternite VIII (figure 9) est doucement allongé et arrondi dans sa partie ventro-apicale, évoquant celui de la sous-espèce nominative (in: Smit, 1975, mais non in: Jordan \& Rothschild, 1913); il ne ressemble pas à celui de l'holotype dessiné par Smith (op. cit., 1966) pour "bemingwayl", mais nous pensons que la membrane apicale de ce segment très fine, est trop fragile pour ne pas se déformer au montage. Le tergite IX (figure 10) correspond parfaitement (aux variations individuelles près) aux dessins de Smith (op. cit., holotype) et de Smit (1975, topotype), avec un sinus, petit mais net, au tiers antérieur du bord supérieur du télomère. On note une infime concavité sur la partie postéro ventrale du basimère chez nos exemplaires. Le sternite IX (figure 10) nous semble unique. Si la portion proximale du sternite est classique, la portion distale est remarquable par sa structure. L'apex est arrondi puis rectiligne, portant peu de soies (six à huit en comptant les soies externes et internes), relativement courtes et très fines. De cet apex part vers le bas une zone membraneuse portant trois soies et une lame ventrale à l'extrémité en "crochet", légèrement sclérotisée. La "membrane" s'étend jusqu'à l'angle, angle fortement 


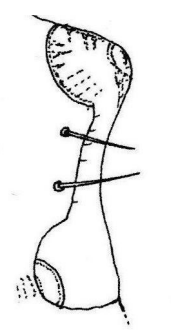

8

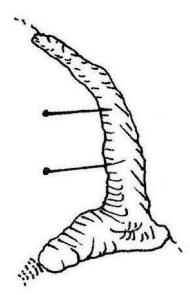

14
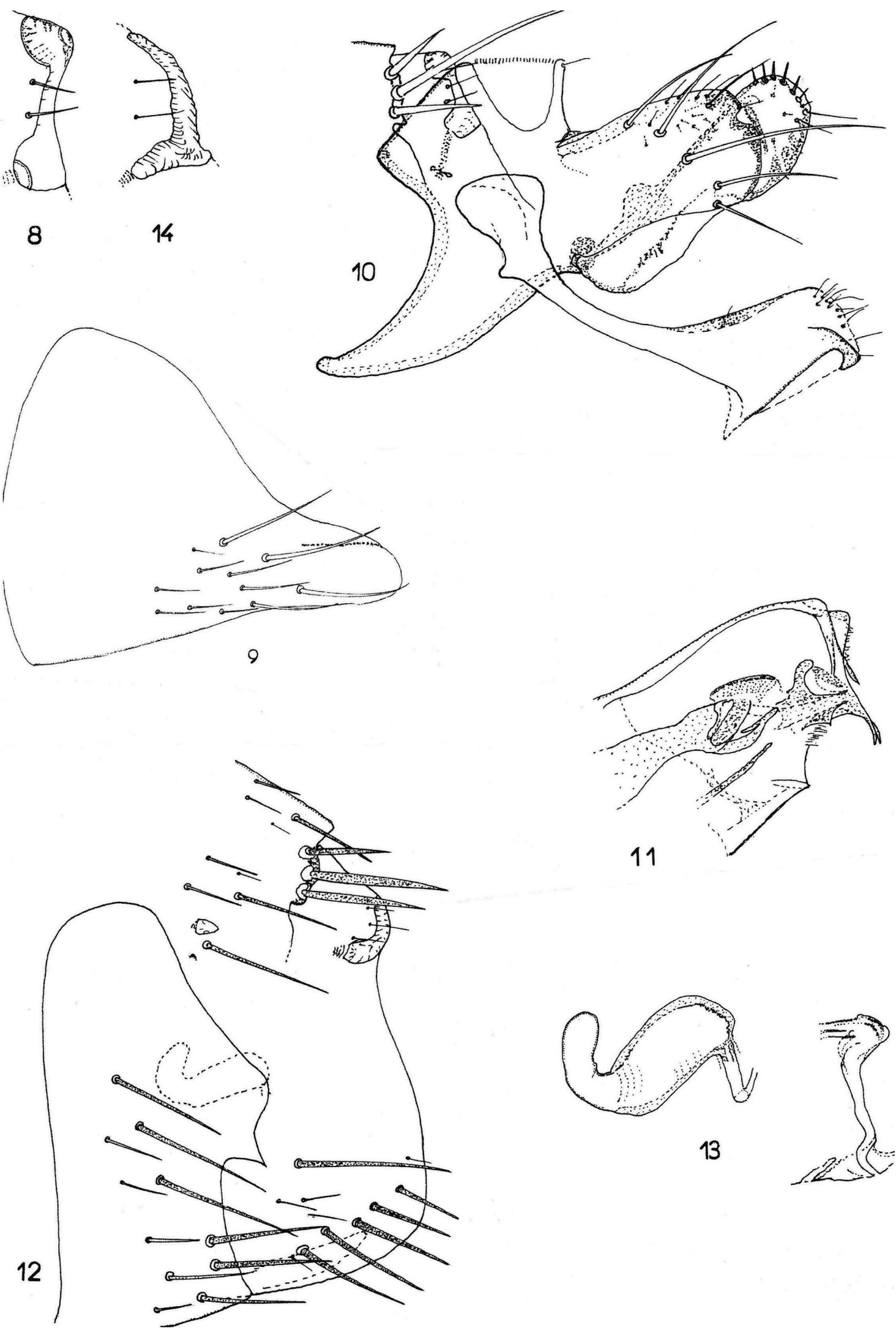

11

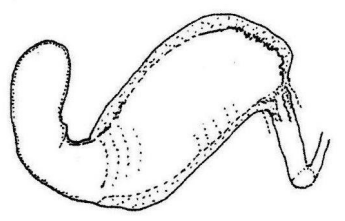

१३

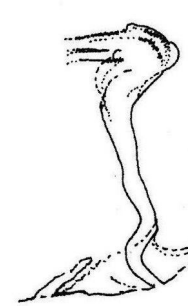

Figs 8-13. - Ctenophthalmus (Ethio.) cophurus hemingwayi Hubbard, 1963. 8 : spiracle du tergite VIII d'une femelle; 9 : sternite VIII d'un mâle; 10 : segment IX; 11 : aedeagus; 12 : sternite VII d'une femelle; 13 : spermathèque et ducti.

Fig. 14. - Ctenophthalmus (Ethio.) kemmelberg Laudisoit \& Beaucournu, 2007b : spiracle du tergite VIII d'une femelle. 
épaissi, formé par les parties proximale et distale du sternite IX. Elle est nettement séparée du bras distal proprement dit par un tracé rectiligne, rétrograde, sclérotisé, surplombant le bord inférieur de cette membrane.

Phallosome (figure 11). L' aedeagus du phallosome de C. c. hemingwayi n'ayant été dessiné que par Smith (op. cit., holotype), toute comparaison est difficile pour les raisons données plus haut, mais il semble assez proche de celui de C. c. schmiederi (holotype, figure 10, in : Smit, 1975). Il s'en sépare au moins par une membrane latérale, donc symétrique, spiculeuse, émanant du lobe dorso-médian, repliée sur elle-même à l'apex de cette pièce et, surtout, par une longue carina sclérotisée sur son bord ventral. Le sclérite de Ford, le tubus interior et l'bamulus sont courts.

Le sternite VII des femelles, comme la spermathèque, ne semblent pas montrer de caractères distinctifs. Toutefois, aucun dessin n'en ayant été donné (sauf par Hubbard, op. cit.), nous le figurons chez un de nos exemplaires (figure 12) : le crochet formé par les sinus de la marge postérieure semble plus accentué que chez C. c. cophurus (cf. Jordan \& Rothschild, 1913), sans atteindre toutefois la forme en "bec de rapace" figurée par Hubbard (1963, 1964).

La spermathèque et les $d u c t i$ (figure 13) ne nous montrent aucun caractère distinctif.

Cette sous-espèce, avec C. c. prolapsus Smit, nous paraît être la plus typée du complexe "cophurus".

\section{REMERCIEMENTS}

I es auteurs remercient l'équipe du Pr R.H. Makundi du Pest Management Centre de la Sokoine University of Agriculture de Morogoro (Tanzanie), l'Université d'Anvers, en particulier le groupe EVOECO, et les Fonds belges pour la Recherche Scientifque (FNRS) et pour la Recherche dans l'Industrie et l'Agriculture (FRIA) pour leur soutien logistique, matériel et financier durant les campagnes de piégeage de rongeurs. À Rennes, Stéphanie Renault est remerciée pour son aide technique.

\section{RÉFÉRENCES}

Beaucournu J.-C. \& Laudisoit A. Signification écologique de la morphologie et de la taille des spiracles chez les Siphonaptères. Bulletin de la Société entomologique de France, 2009, 114, 63-68.

Hopkins G.H.E. \& Rothschild M. An illustrated Catalogue of the Rothschild Collection of fleas (Siphonaptera) in the British Museum (Natural History). Vol. IV Hystrichopsyllidae. Trustees of the British Museum (Nat. Hist.), London, 1966, 549 p., 12 pl.
HubBard C.A. A dozen new Fleas from Tanganyika. East African Medical Journal, 1963, 40, 408-419.

HubBard C.A. A Flea named for Michael Grzimek. Entomological News, 1964, 25, 155-158.

HubBard C.A. Ctenophthalmus cophurus schmiederi n. ssp. (Siphonaptera). Entomological news, 1966, 77, 100-103.

JORDAN K. \& ROTHSCHILD N.C. Siphonaptera collected by $\mathrm{Mr}$ Robin Kemp in tropical Africa. Novitates Zoologicae, 1913, 20, 528-581.

Laudisoit A. \& Beaucournu J.-C. Deux nouveaux Ctenophthalmus (Insecta : Siphonaptera : Ctenophthalmidae) de Tanzanie. Parasite, 2007a, 14, 101-105.

Laudisoit A. \& Beaucournu J.-C. Ctenophthalmus (Ethioctenophthalmus) kemmelberg n. sp. (Insecta : Siphonaptera : Ctenophthalmidae), Puce nouvelle de Tanzanie et description de structures internes non signalées chez les Mécoptèroïdes. Parasite, 2007b, 14, 213-217.

Smit F.G.A.M. Species-groups in Ctenophthalmus (Siphonaptera : Hystrichopsyllidae). Bulletin of the British Museum (Natural History), Entomology, 1963, 14, 107-152, 58 pl.

SMIT F.G.A.M. Siphonaptera from the Cherangani Hills in Kenya. Revue de Zoologie Africaine, 1975, 89, 191-210.

Verheyen W.N., Hulselmans J.L., Dierckx T., Mulungu L., LeIRS H., CORTI M. \& Verheyen E. The characterization of the Kilimanjaro Lophuromys aquilus True 1892 population and the description of five new Lophuromys species (Rodentia: Muridae). Bulletin de l'Institut royal des Sciences naturelles de Belgique: biologie, 2007, 77, 23-76.

Venchenko L.A., Verheyen W.N., Verheyen E., Hulselmans J. \& LeIRs H. Morphometric and genetic study of Ethiopian Lophuromys flavopunctatus THOMAS, 1888 species complex with description of three new 70-chromosal species (Muridae, Rodentia), Bulletin de l'Institut royal des Sciences naturelles de Belgique : biologie, 2007, 77, 77-118.

Wilson D.E. \& ReEDer D.M. Mammal species of the World; a taxonomic and geographic reference. Smithsonian Institution Press, second edition, 1992, XVI + 1207 p.

Reçu le 6 mai 2009

Accepté le 27 juillet 2009 